\title{
Estrategias de aprendizaje para fortalecer el rendimiento académico en matemática del bachillerato de la unidad educativa el empalme, Ecuador
}

\author{
Manuel Bermúdez-Pacheco \\ p7001266895@ucvvirtual.edu.pe \\ https://orcid./0000-0003-3582-0079 \\ maberpa10@hotmail.com \\ Milton Cayambe-Guachilema \\ p7001262556@ucvvirtual.edu.pe \\ https://orcid./0000-0003-2298-7697 \\ cayambe1970@hotmail.com \\ Gardenia Gómez-Samaniego \\ p7001262519@ucvvirtual.edu.pe \\ https://orcid./0000-0003-2298-7697 \\ unidadeducativafiscalvr@gmail.com \\ Carlos Nuñez-Michuy \\ cnunesz@ueb.edu.pe \\ https://orcid./0000-0003-2298-7697 \\ Universidad César Vallejo \\ Piura- Perú
} RESUMEN

La presente investigación estrategias de aprendizaje para fortalecer el rendimiento académico en matemática de los estudiantes del bachillerato de la Unidad Educativa El Empalme, del Ecuador. Tuvo como objetivo Proponer un programa de estrategias de aprendizaje para fortalecer el rendimiento académico en matemática de los estudiantes del bachillerato de la Unidad Educativa El Empalme, Ecuador. Esta investigación utilizó enfoque cuantitativo, de tipo básica o fundamental, con diseño no experimental, descriptiva- propositiva, se trabajó con una muestra de 34 estudiantes los mismos que fueron elegidos con un muestreo no probabilístico, a criterio del investigador; como instrumento para el recojo de los datos se utilizó el cuestionario. Asimismo, de acuerdo a los resultados para la variable rendimiento académico en matemática tenemos que un $56 \%$ de los estudiantes no alcanza los aprendizajes requeridos, asimismo un $26 \%$ están próximo a alcanzar los aprendizajes requeridos, finalmente un $18 \%$ se evidencia que alcanzan los aprendizajes requeridos. En este sentido se planteó la propuesta de estrategias de aprendizaje la misma que fue validada por jueces expertos en el tema.

Palabras clave: aprendizaje; estrategias; matemática; rendimiento; razonamiento 


\title{
Learning strategies to strengthen academic performance in mathematics at the el empalme educational unit, Ecuador
}

\begin{abstract}
The present investigation learning strategies to strengthen the academic performance in mathematics of the high school students of the El Empalme Educational Unit, of Ecuador. Its objective was to propose a program of learning strategies to strengthen the academic performance in mathematics of high school students from the El Empalme Educational Unit, Ecuador. This research used a quantitative approach, of a basic or fundamental type, with a non-experimental, descriptive-propositional design. We worked with a sample of 34 students, who were chosen with a non-probabilistic sampling, at the discretion of the researcher; The questionnaire was used as an instrument for data collection. Likewise, according to the results for the variable academic performance in mathematics we have that $56 \%$ of the students do not reach the required learning, also $26 \%$ are close to achieving the required learning, finally $18 \%$ it is evidence that they achieve the learning required. In this sense, the proposal of learning strategies was proposed, which was validated by expert judges on the subject.
\end{abstract}

Key words: learning; strategies; mathematics; performance; reasoning

Artículo recibido: 02 Setiembre. 2021 Aceptado para publicación: 30 Setiembre. 2021 Correspondencia: maberpa10@ hotmail.com Conflictos de Interés: Ninguna que declarar 


\section{INTRODUCCIÓN}

Actualmente, a través de un lenguaje cotidiano denominamos ansiedad matemática a la falta de razonamiento; eso no significa que consideremos que los números irracionales son irrazonables. Pero lamentablemente existe un temor en muchos estudiantes por comprender, comunicar y resolver los números en sus diversas manifestaciones, lo que genera relativamente que los niveles de rendimiento en matemática se vean afectados por tal manifestación y que algunos denominan aritmofobia.

Según Bolívar (2021) la aritmofobia se denomina al temor colosal e ilógico desligando por la representación o adelanto a un objeto o a un contexto, por ejemplo: el mostrar el libro de matemática, anticipar que tendremos exposición de números impares, anticipar que mañana habrá clase de la asignatura geometría, el estar ante un examen de matemática. Las investigaciones indican que frente a las fobias específicas la genética contribuye moderadamente en el desarrollo de miedos irracionales, por lo tanto, en este miedo específico la mayor influencia se debe a factores ambientales, es decir, las experiencias, episodios, situaciones traumáticas que pueden haber vivido en torno a matemática.

Por lo descrito en el acápite anterior tenemos que su argumento se fundamenta en los resultados más recientes de las pruebas PISA 2018, en los que Colombia alcanzo porcentajes muy inferiores a lo que representa la Organización para la Cooperación y el Desarrollo Económicos (Ocde) en matemática. "Los estudiantes colombianos en un 35\% se ubicaron en el nivel dos o superior, mientras en todos los países de este organismo la media fue del $76 \%$ de los estudiantes alcanzando por lo menos este nivel de competencia en matemática”. (Bolívar, 2021, p.34).

Asimismo, tenemos que en España en la última edición de las tendencias en matemáticas y ciencias la misma que trata de evaluar a niños de los primeros grados en formación, en este sentido los estudiantes españoles lograron un puntaje de 502 en el dominio de las ciencias matemáticas en relación a los 527 puntos logrados por los países de la OCDE, incluso sobre los 513 puntos obtenidos por la UE. El problema de acuerdo a la investigación es que nunca hemos llegado a sobresalir en matemática y así lo reafirman los resultados detallados en el informe PISA. Por ejemplo: en la edición 2018, los estudiantes españoles de 15 años obtuvieron el calificativo más bajo en ciencias desde que se aplicó las prueba PISA. 
Por otro lado, en el Ecuador se evidencia un gran problema en los estudiantes al finalizar el año escolar, en relación al nivel de rendimiento académico siendo bajo en el dominio de matemática y en consecuencia la no aprobación de la materia, esta dificultad se evidencia en la mayoría de colegios, los porcentajes de rendimiento académico son publicados todos los años a través de diferentes medios de comunicación, especialmente el nivel de bajo rendimiento y la media aritmética no muy satisfactoria alcanzada en función al aprendizaje de las ciencias matemáticas. En este sentido, se presentan dificultades grandes y graves de los alumnos ecuatorianos para actuar de forma competente en contextos que movilizan la resolución de problemas matemáticos de acuerdo a los resultados de las evaluaciones internacionales PISA-D 2018, en las que por primera vez participó Ecuador. De acuerdo a estos resultados tenemos que el 70,8\% de los alumnos ecuatorianos no lograron el nivel 2 en Matemáticas, es decir no logró superar la categoría del nivel de desempeño básico. (Diario el Universo, 2019).

Frente a esta problemática identificada la investigación buscó solucionar a través de la propuesta de estrategias de aprendizaje, en donde surge la necesidad de fortalecer y mejorar el aprendizaje de las ciencias matemáticas, teniendo en cuenta que existen metodologías tradicionales y que implica que algunas estrategias, método y técnicas de aprendizaje que se necesitan sean desplazadas por otras más activas e innovadoras en el proceso de aprendizaje, y sobre todo nos permitirá diseñar otras alternativas que permitan al estudiante interactuar de manera más dinámica, activa que permitan desarrollar técnicas o estrategias en donde el estudiantes se compromete en su aprendizaje, en donde la Unidad Educativa El Empalme no es ajena a esta problemática.

De acuerdo al párrafo anterior en la Unidad Educativa El Empalme, en la provincia del Guayas-Ecuador, una población de estudiantes consultados, consideraron que, la asignatura de matemática les resulta complicadas de entenderlas, comprenderlas y aprenderlas, además de observarse docentes que no son de la especialidad, con escasos recursos didácticos que no generan motivación pedagógica, así como, una deficiente aplicación de estrategias para el aprendizaje, dando como resultado un entorno de desmotivación y desinterés estudiantil por asimilar los conocimientos sobre la asignatura antes mencionada.

Frente a esta situación, es necesario formular el problema de investigación, que permita direccionar el estudio sobre la temática a ser investigada, para ello se redactó la siguiente 
pregunta de investigación: ¿De qué manera las estrategias de aprendizaje fortalece el rendimiento académico en matemáticas de los estudiantes del bachillerato de la Unidad Educativa El Empalme, Ecuador-2020?

El estudio se justifica teóricamente porque analiza el comportamiento del problema y fundamenta alternativa de solución, esta información puede ser consultada por futuros estudios sobre temas relacionados. Su justificación práctica se sustenta en que aporta en resolver la problemática sobre el aprendizaje de matemática en los estudiantes del plantel. $\mathrm{Su}$ justificación metodológica, se centra en la forma de adquirir información real, contrastar resultados, validar instrumentos de acopio de datos y asegurar el contenido de la propuesta como una experiencia exitosa.

Por consecuencia el objetivo fue Proponer estrategias de aprendizaje para fortalecer el rendimiento académico en matemática de los estudiantes del bachillerato de la Unidad Educativa El Empalme, Ecuador-2020.

\section{METODOLOGÍA}

La presente investigación estuvo orientada a desarrollar investigación básica, el cual para Sánchez y Reyes (1998) es la investigación que se orienta al acopio de datos reales con la finalidad de aportar al conocimiento científico, para descubrir temas de convergencia" (p.56). Cabe mencionar a Caballero (2014) quien sostiene que un estudio descriptivo está condicionado a una posesión cuantitativa, el cual hace uso de métodos precisos y métodos narrativos permitiendo la determinación de los fenómenos observados" (p.100).

Por otro lado, de acuerdo a las características de la investigación también se centra en el tipo propositivo, es por ello que según Hurtado de Barrera (2008) indica que una investigación propositiva o prospectiva pretende dar alternativas de solución a algunas situaciones específicas, la cual parten de la exploración. Esta alternativa da soluciones previas a un diagnóstico de la realidad, buscando la innovación, sin embargo, no siempre serán realizadas (p.100). Cabe indicar que en esta investigación se diagnosticó la variable rendimiento matemático y con ello se diseñó la propuesta sobre estrategias de aprendizaje de las matemáticas. 


\section{Tabla 1}

Distribución de la muestra en base al género.

\begin{tabular}{lcc}
\hline \multicolumn{1}{c}{ Muestra } & F & \% \\
\hline Varones & 21 & 62 \\
Mujeres & 13 & 38 \\
Total & 34 & 100 \\
\hline
\end{tabular}

Fuente: Rectoría de institución educativa en El Empalme, 2020.

$\mathrm{Si}$ bien el procedimiento de todo proceso de investigación, sintetizando los planteamientos de Ander-Egg (2011), incluye tanto las actividades de trabajo de campo, como las de trabajo de gabinete y constituyeron desde la problematización hasta las conclusiones del estudio; se detalla el procedimiento seguido en la recolección de información en la siguiente figura.

\section{Figura 1}

Procedimientos de investigación

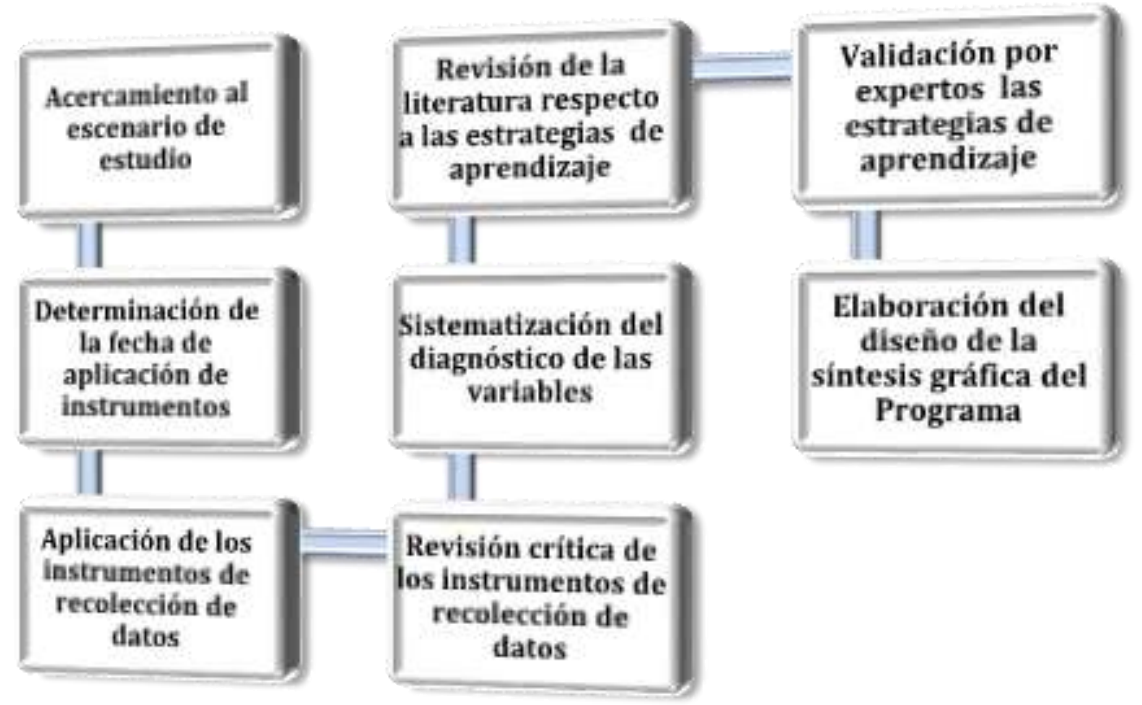

Fuente: Elaboración propia.

\section{RESULTADOS}

Los datos obtenidos mediante la aplicación virtual del instrumento se muestran en la siguiente tabla. 


\section{Tabla 2}

Resumen de la variable rendimiento matemático

\begin{tabular}{lcccccccc}
\hline \multirow{2}{*}{ Calificativos } & \multicolumn{2}{c}{$\begin{array}{c}\text { Razonamiento y } \\
\text { Demostración }\end{array}$} & \multicolumn{2}{c}{$\begin{array}{c}\text { Comunicación } \\
\text { Matemática }\end{array}$} & $\begin{array}{c}\text { Resolución de } \\
\text { Problemas }\end{array}$ & \multicolumn{2}{c}{$\begin{array}{c}\text { Rendimiento } \\
\text { Matemático }\end{array}$} \\
\cline { 2 - 9 } & $\mathbf{N}^{\circ}$ & $\mathbf{\%}$ & $\mathbf{N}^{\circ}$ & $\mathbf{\%}$ & $\mathbf{N}^{\circ}$ & $\mathbf{\%}$ & $\mathbf{N}^{\circ}$ & $\%$ \\
\hline$[00.0-4.99]$ & 17 & 50 & 13 & 38 & 22 & 64 & 19 & 56 \\
{$[5.00-6.99]$} & 11 & 32 & 16 & 47 & 7 & 21 & 9 & 26 \\
{$[7.00-8.99]$} & 6 & 18 & 5 & 15 & 5 & 15 & 6 & 18 \\
{$[9.00-10.00]$} & 00 & 0 & 00 & 0 & 0 & 0 & 0 & 0 \\
TOTAL & 34 & 100 & 34 & 100 & 34 & 100 & 34 & 100 \\
\hline \multicolumn{1}{c}{ Promedio } & $\mathbf{4 . 7}$ & \multicolumn{3}{c}{$\mathbf{5 . 3}$} & & $\mathbf{3 . 3}$ & & $\mathbf{4 . 4}$ \\
\hline
\end{tabular}

Fuente: Evaluación a estudiantes.

En relación a la Tabla 2 se pone a consideración el resumen de la variable rendimiento matemático y sus dimensiones, las mismas que han sido desarrolladas de forma progresiva. Es importante manifestar que los calificativos que oscilan entre 00 a 4.99 según lo señalado por el Ministerio de Educación implica que el estudiante "no alcanza los aprendizajes requeridos (suspendido Automáticamente)", los mismo los calificativos que oscilan desde el 6.99 - 5.00, corresponde a que "está próximo a alcanzar los aprendizajes requeridos; por otro lado los calificativos desde 8.99 - 7.00, significa que se "alcanza los aprendizajes requeridos, finalmente los calificativos que oscilan entre las notas de 10.00 - 9.00 significa que "domina los aprendizajes requeridos".

En relación a la propuesta de estrategias de aprendizaje de las matemáticas se ha considerado los principios que rigen la propuesta como el principio de igualdad y principio tecnológico, cuyas características son facilidad en el acceso, disponibilidad de información, posibilidades comunicativas y manejo de lo digital, sustentado en las teorías del aprendizaje significativo, cognitivismo, conductismo, teoría del aprendizaje por descubrimiento, teoría de Dienes y el modelo de Van Hiele, además de las bases epistemológica, filosóficas, psicológicas y pedagógicas que la sustentan. Este conjunto de estrategias ha sido agrupado en estrategias para activar (o generar) conocimientos previos en donde se considera lluvia de ideas y casuísticas, además de estrategias para orientar la atención -concentración, en la cual encontramos: cuadros mágicos, crucigrama matemático, rompecabezas y Juegos de memoria; finalmente las estrategias para 
organizar la información en donde destacan la tabla pitagórica, bingos de combinaciones y resultados

Después de haber diagnosticado la variable aprendizaje de las matemáticas se hizo necesario la elaboración de la propuesta tal como se presenta en la figura 2, para ello tiene que cumplir con el criterio de validarla. Por lo que se consideró varios aspectos que deben ser evaluados, como la redacción científica, lógica de la investigación, problema de estudio, objetivos de la investigación, previsiones metodológicas, fundamentación teórica y epistemológica, bibliografías, anexos, fundamentación y viabilidad de modelo, fundamentación y viabilidad de los instrumentos; con las categorías de muy adecuado (MA), bastante adecuado (BA), adecuado (A), poco adecuado (PA) y no adecuado (NA). En este sentido se validó la propuesta por expertos en el tema, en la cual después de leer cada uno de los indicadores se determinó que la propuesta presenta coherencia con lo solicitado en el formato de los expertos.

\section{Figura 2}

Teorías, principios y fundamentos de la Propuesta de estrategias de aprendizaje

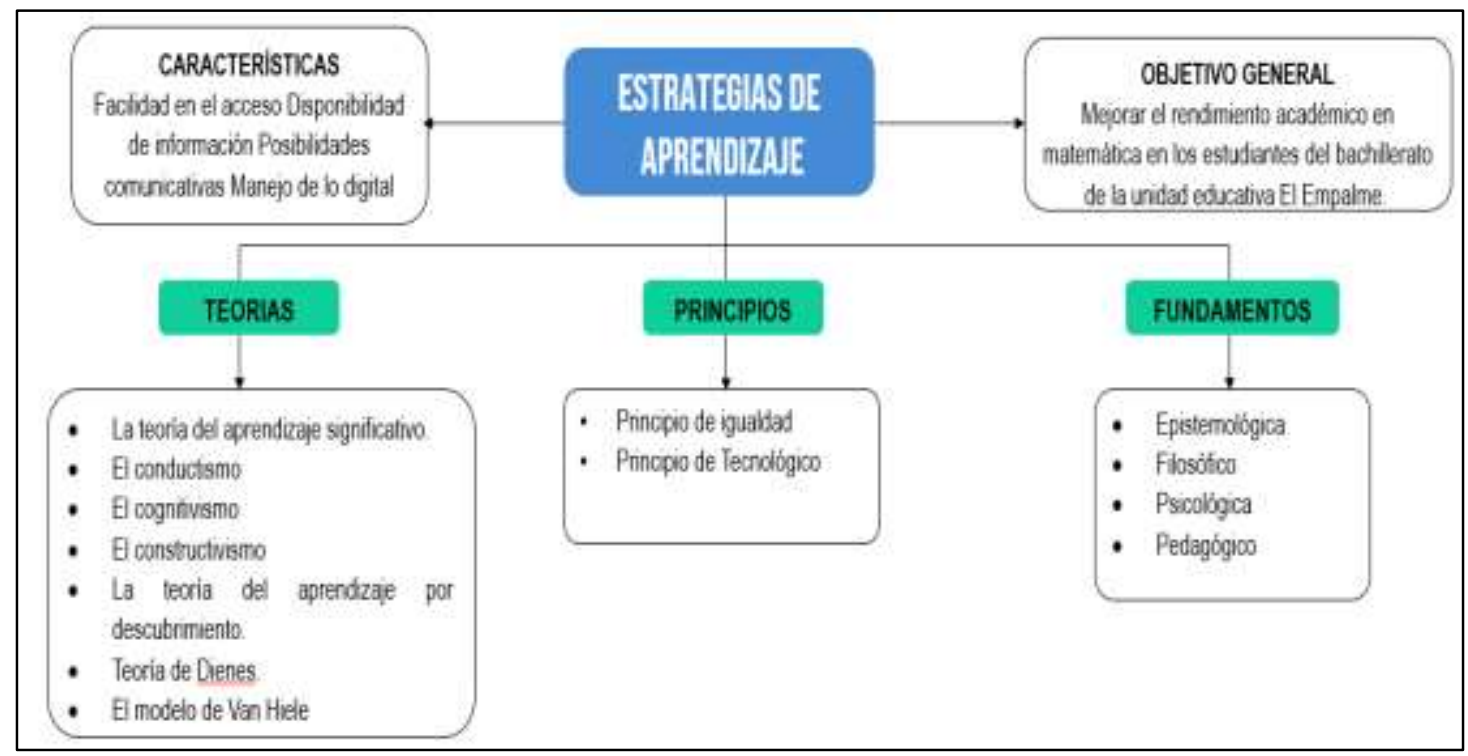

\section{DISCUSIÓN}

De acuerdo al objetivo específico relacionado a diagnosticar la variable rendimiento académico en matemática, se hace necesario abordarlo por dimensiones para un mejor entendimiento: en este sentido la dimensión razonamiento y demostración la misma que se considera como un proceso de inferir, de establecer correspondencia, relaciones en una serie o secuencia finita de fórmulas tales que cada una es un axioma o una consecuencia 
inmediata de algunas fórmulas precedentes, es por ello que los estudiantes solucionan los problemas u obtienen información debido a los procesos de razonamiento. Delgado, Espinoza (2019). De acuerdo a lo planteado tenemos que en la Tabla 2 se evidencia 50\% de los estudiantes que no alcanza los aprendizajes requeridos (suspendido Automáticamente), inclusive encontramos un $32 \%$ que indica que "está próximo a alcanzar los aprendizajes requeridos", además de un $18 \%$ se evidencia que alcanzan los aprendizajes requeridos. De estos resultados se discrepa con Gonzáles (2016) en el cual concluye en su investigación que existen bajos calificativos y están en relación a las edades de los participantes, en tal sentido el rendimiento académico en matemática es bajo. Por lo descrito anteriormente se puede inducir que a pesar de utilizar estrategias de aprendizaje y también enseñanza persiste y es un común en muchas instituciones educativas el problema del aprendizaje de los estudiantes; asimismo esta dimensión es muy importante en el desarrollo de las matemáticas toda vez que permite actuar con mayor eficacia en la toma de decisiones y en numerosos aspectos de la vida cotidiana. También es oportuno indicar que el hecho que se enseñe matemáticas en las instituciones educativas responde a una necesidad a la vez individual y social; esas necesidades matemáticas que surgen y fomentan que el aprendiz aplique la información a una mayor variedad de contextos, espacios, situaciones, hechos, continuar cadenas argumentales, identificar la idea o ideas principales, estimar y enjuiciar la lógica y validez de argumentaciones e informaciones.

Para la dimensión comunicación matemática que es entendida como la organización y comunicación del pensamiento matemático con claridad, relación y coherencia; expresando ideas matemáticas con exactitud; reconociendo conexiones entre conceptos, definiciones matemáticas y la realidad, y aplicándolos a hechos, situaciones, contextos verídicos. (Delgado \& Zakaryan, 2020) asimismo es entendida, como el transcurso a través del cual se alcanza una lógica integradora comunicativa mediante la interacción entre todos los sujetos socializadores del proceso matemático formativo, que posibilita el compartir la diversidad de los símbolos matemáticos (Alfaro et al., 2019). En este sentido en relación a los resultados en la Tabla 2 tenemos que la dimensión comunicación matemática presenta un $38 \%$ de los estudiantes tienen calificativos muy bajos por lo tanto no alcanzan los aprendizajes requeridos, asimismo el $47 \%$ está próximo a alcanzar los aprendizajes requeridos incluso un $15 \%$ se evidencia que alcanzan los aprendizajes 
requeridos. Estos resultados permiten coincidir con Landeta (2017) en la cual sostiene que existe un $47 \%$ de estudiantes que sus calificativos son regulares es decir que han aprobado con promedios entre 11 y 14; asimismo encontramos un escaso $23 \%$ de estudiantes que tienen notas sobresalientes; además de un preocupante $30 \%$ que se encuentran con notas por debajo de 10 es decir están desaprobados. De acuerdo a estos resultados podemos inducir que los estudiantes es muy difícil que sientan la necesidad de estudiar matemáticas, incluso entre personal consideradas socialmente cultas se agotan, en este sentido la falta de ganas de estudiar.

En relación a la dimensión resolución de problemas teóricamente se puede entender como está directamente relacionada a fomentar que el estudiante construya nuevos aprendizajes previa resolución de problemas de un contexto real o matemático; permitiéndole que tenga oportunidades de aplicar y adaptar diferentes estrategias en diversos contextos, situaciones, hechos, para controlar el proceso de solución del problema debe reflexionar sobre este y sus resultados. (Zaslavsky \& Shir, 2015); asimismo la resolución de problemas indica que es fundamental que se brinde la oportunidad de aplicar diversas herramientas en la medida que el estudiante busque solucionar los problemas en diversos contextos, admitiendo la interacción con otras disciplinas, las mismas que permiten consolidar el desarrollo de competencias para hacer frente a situaciones problemáticas dentro del campo de la disciplina matemática. En su sentido más amplio Climent (2005) sostiene que la resolución de un problema comienza con la identificación del inconveniente en cuestión. Después de todo, si no se tiene conocimiento sobre la existencia de la contrariedad o no se la logra determinar con precisión, no habrá tampoco necesidad de encontrar una solución. Por lo manifestado de acuerdo a la Tabla 2 tenemos que relacionado a la dimensión resolución de problemas encontramos un $64 \%$ de estudiantes que no alcanza los aprendizajes requeridos, por otro lado, encontramos un $27 \%$ de los evaluados que se ubican en el criterio de que "está próximo a alcanzar los aprendizajes requeridos", asimismo un escaso $15 \%$ se evidencia que alcanzan los aprendizajes requeridos. De acuerdo a lo manifestado se puede concluir que esta dimensión es la más compleja toda vez que es más técnica y operativa, es por ello ante la ausencia de una verdadera disciplina matemática la falta de motivación y buena actitud hacia las matemáticas se convierten en dificultades para empezar a estudiar, es por ello 
que muchas veces es cuestión de orden y compromiso para desarrollar una buena práctica en el desarrollo del conocimiento de las matemáticas.

En lo que se refiere a la variable rendimiento matemático se asume que teóricamente se considera o se conceptualiza por parte de Ochoa (2011) quien la define a la matemática como la ciencia que estudia estructuras, conjuntos, espacios, tiempo, correspondencia y el cambio. La matemática se fundamenta en axiomas y teoremas ya demostrados deduciendo cada conjetura aceptada. En función a los resultados que se presentan en la Tabla 2 se evidencia que la variable rendimiento académico en matemática tenemos un $56 \%$ de los estudiantes que no alcanza los aprendizajes requeridos, por otro lado, encontramos un $26 \%$ de los estudiantes evaluados que se ubican en el criterio de que "está próximo a alcanzar los aprendizajes requeridos". Asimismo, el $18 \%$ consideran que alcanzan los aprendizajes requeridos. Estos resultados discrepan con Guzmán (2019) en el cual en su investigación se obtuvieron resultados descriptivos resaltando que el 85\% de los estudiantes demostraron que el razonamiento lógico matemático ha influido significativamente en el rendimiento académico de Matemática I y la influencia fue baja en un $15 \%$. Frente a ello se puede determinar que los resultados de diversas investigaciones y experiencias han demostrado la forma de apoyo en puntos concretos debiendo ser guiados por el juicio y la actividad pedagógica. Los docentes deben conocer y comprender con profundidad la matemática que enseñan y tener la capacidad de apoyarse en ese conocimiento con flexibilidad en sus tareas docentes logrando la eficacia en la enseñanza de matemática, en ese sentido nace la propuesta sobre estrategias de aprendizaje.

En lo que corresponde a la propuesta de estrategias de aprendizaje debe entender como el conjunto de habilidades, pasos, experiencias que un alumno obtiene, logra y utiliza de forma voluntaria como instrumento, herramienta flexible promoviendo un aprendizaje significativo y solucionando dificultades, problemas, demandas y exigencias académicas con Díaz, Hernández (2002). Es oportuno manifestar la tarea de las instituciones educativas y en particular de los docentes en donde se busca la forma de crear las mejores condiciones posibles para que los estudiantes puedan aprender, destacando los contenidos que les son útil en su vida diaria. En este sentido nace la propuesta en mención sobre la disciplina matemática y que requiere una interrelación muy estrecha entre la utilización 
de técnicas e inclusive la tecnología que permita justificar la actividad matemática y presentarlo como algo comprensible para quienes quieran estudiarla.

En el paradigma de las estrategias de aprendizaje sobre todo para la matemática es importante indicar tres aspectos indisolubles: utilizar matemáticas conocidas, aprender y enseñar matemáticas y crear matemáticas nuevas; en este sentido siguiendo el proceso de estudio, cuando se dispone de una formulación precisa de algunos problemas relativas a la enseñanza y aprendizaje de las matemáticas es fundamental abordar la didáctica para tener en cuenta un mejor tratamiento de los recursos, técnicas y procedimientos dentro del marco de las estrategias de aprendizaje. Por otro lado, la propuesta busca desarrollar un conjunto de dificultades para la enseñanza - aprendizaje de esta disciplina, lógicamente requiere del compromiso de los maestros para poder aplicar dicha propuesta y buscar paulatinamente la superación académica.

\section{CONCLUSIONES}

- Producto de los resultados la dimensión razonamiento y demostración nos indica que un $50 \%$ de los estudiantes no alcanza los aprendizajes requeridos, por otro lado, encontramos $32 \%$ de los evaluados que se ubican en el criterio de que "está próximo a lograr los aprendizajes requeridos", finalmente un $18 \%$ se evidencia que alcanzan los aprendizajes requeridos.

- La dimensión comunicación matemática se evidencia que el 38\% de los estudiantes que no alcanza los aprendizajes requeridos, asimismo un $47 \%$ indican que "está próximo a alcanzar los aprendizajes requeridos", además de un escaso $15 \%$ se evidencia que alcanzan los aprendizajes requeridos.

- La dimensión resolución de problemas encontramos un $64 \%$ de los estudiantes no consiguen los aprendizajes requeridos, por otro lado, encontramos un $27 \%$ de los evaluados que se ubican en el criterio de que "está próximo a alcanzar los aprendizajes requeridos", incluso el 15\% se evidencia que alcanzan los aprendizajes requeridos.

- Para la variable rendimiento académico en matemática se tiene un 56\% de los estudiantes no logra los aprendizajes requeridos, asimismo un $26 \%$ están próximo a alcanzar los aprendizajes requeridos, finalmente un $18 \%$ se evidencia que alcanzan los aprendizajes requeridos. 
- En relación a la propuesta se puede indicar que fue validada por expertos en la cual indican que presenta coherencia y puede ser aplicada en cualquier contexto educativo, en tal sentido es válida.

\section{REFERENCIAS}

Alfaro, C., Flores, P., y Valverde, G. (2019). La demostración matemática: significado, tipos, funciones atribuidas y relevancia en el conocimiento profesional de los profesores de matemáticas. Uniciencia, 33(2), 55-75.

Ander-Egg, E. (2011). Aprender a investigar: nociones básicas para la investigación social. - 1a ed.- Córdoba: Brujas, 2011.

Bolívar, P. (2021, Junio 14). Aritmofobia, un miedo que no se tiene calculado. El Tiempo. https://www.eltiempo.com/vida/ciencia/que-es-la-aritmofobia-o-el-miedo-a-lasmatematicas-595382

Caballero, A. (2014). Metodología integral innovadora para planes y tesis. La Metodología del cómo formularlos. México.

Climent, N. (2005). El desarrollo profesional del maestro de primaria respecto de la enseñanza de la matemática. Un estudio de caso. (Tesis doctoral, Universidad de Huelva). http://rabida.uhu.es/dspace/handle/10272/2742

Delgado, R. y Espinoza, G. (2019). El conocimiento del profesor de Matemáticas sobre la demostración y sus roles en la enseñanza de las Matemáticas. https://www.researchgate.net/publication/339080087_El_Conocimiento_Del_ Profesor_De_Matematicas_Sobre_La_Demostracion_Y_Sus_Roles_En_La_ Ensenanza_De_Las_Matematicas

Delgado, R., Zakaryan, D. (2020). Relationships between the knowledge of practices in mathematics and the pedagogical content knowledge of a mathematics lecturer. International Journal of Science and Mathematics Education, 18(3), 567-587. https://ouci.dntb.gov.ua/works/4g6vvj37/

Diario el Universo (2019, febrero 26). Ecuador reprobó en Matemáticas en evaluación internacional.

https://www.eluniverso.com/guayaquil/2019/02/26/nota/7207946/matematicasno-se-paso-prueba/

Díaz, F. y Hernández, G. (2002). Estrategias docentes para un aprendizaje significativo: una interpretación constructivista. Mc Graw Hill. México 
Gonzáles, D. (2016). Relación entre el rendimiento académico en matemáticas y variables afectivas y cognitivas en estudiantes preuniversitarios de la universidad católica Santo Toribio de Mogrovejo. (Tesis doctoral, Universidad de Málaga). https://riuma.uma.es/xmlui/bitstream/handle/11630/11691/TD_GONZALES_L OPEZ_David_Ysrael.pdf?sequence=1\&isAllowed=y

Guzmán, P. (2019). El razonamiento lógico matemático y su influencia en el rendimiento académico en Matemática I de los estudiantes del primer ciclo de una Universidad Privada, 2018. (Tesis de doctorado, Universidad Nacional de $\begin{array}{llll}\text { Educación } & \text { Enrique } & \text { Guzmán } & \text { y }\end{array}$ https://repositorio.une.edu.pe/bitstream/handle/UNE/3044/TM\%20CEEm\%204409\%20P1\%20$\% 20$ Paulino\%20Jimenez\%20Guzman\%20David.pdf?sequence=1\&isAllowed $=\mathrm{y}$

Hurtado de Barrera, J. (2008). Metodología de la investigación, una comprensión holística. Investigación proyectiva. http://investigacionholistica.blogspot.com/2008/02/la-investigacinproyectiva.html

Landeta, L. (2017). La motivación y el rendimiento académico en las materias de Matemáticas y estadística. (Tesis doctoral, Universidad de Alicante). https://rua.ua.es/dspace/bitstream/10045/85907/1/tesis_\%20luis_gonzalo_landet a_bejarano.pdf

Ochoa, G. (2011). Motivación en el proceso de enseñanza aprendizaje de la matemática. (Tesis de grado, Universidad Estatal de Milagro). http://repositorio.unemi.edu.ec/bitstream/123456789/1921/1/Motivaci\%C3\%B3 n\%20en\%20e1\%20proceso\%20de\%20ense\%C3\%B1 anza\%20aprendizaje\%20de $\% 201 \mathrm{a} \% 20$ matem\%C3\%A1tica.pdf

Sánchez, H., Reyes, C. (1998). Metodología y diseño en la investigación científica. Primera reimpresión. Lima -Perú. Editorial Mantaro.

Zaslavsky, O., y Shir, K. (2015). Students' conceptions of a mathematical definition. Journal for Research in Mathematics Education, 36(4), 317-346. https://www.jstor.org/stable/30035043 